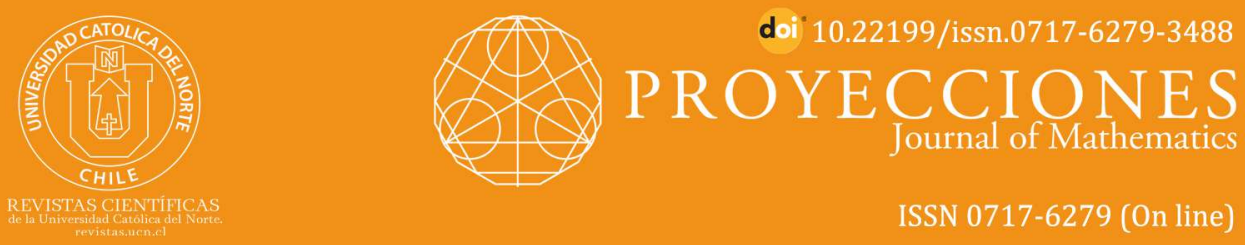

\title{
Lyapunov-type inequality for a Riemann-Liou- ville type fractional boundary value problem with anti-periodic boundary conditions
}

\section{Jagan Mohan Jonnalagadda1ำ orcid.org/0000-0002-1310-8323}

Debananda Basua2 ${ }^{2}$ (1) orcid.org/0000-0002-8062-319X

Birla Institute of Technology and Science Pilani, Dept. of Mathematics, Hyderabad, TG, India. 1⿴j.jaganmohan@hotmail.com; ${ }^{2}$ p20170428@hyderabad.bits-pilani.ac.in

Received: 2019/04/19 | Accepted: 2021/01/28

\section{Abstract:}

In this article, we establish a Lyapunov-type inequality for a two-point Riemann-Liouville type fractional boundary value problem associated with well-posed anti-periodic boundary conditions. As an application, we estimate a lower bound for the eigenvalue of the corresponding fractional eigenvalue problem.

Keywords: Riemann-Liouville type fractional derivative; Boundary value problem; Green's function; Lyapunov inequality; Eigenvalue estimate.

MSC (2020): 34A08, 34A40, 26D10, 33E12, 34C10.

\section{Cite this article as (IEEE citation style):}

J. M. Jonnalagadda and D. Basua, "Lyapunov-type inequality for a Riemann-Liouville type fractional boundary value problem with antiperiodic boundary conditions", Proyecciones (Antofagasta, On line), vol. 40, no. 4, pp. 873-884, 2021, doi: 10.22199/issn.0717-6279-3488

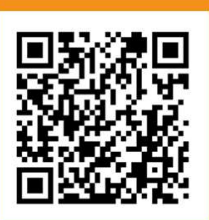

Article copyright: (C) 2021 Jagan Mohan Jonnalagadda and Debananda Basua. This is an open access article distributed under the terms of the Creative Commons License, which permits unrestricted use and distribution provided the original author and source are credited. 


\section{Introduction}

In 1907, Lyapunov [6] proved a necessary condition for the existence of a nontrivial solution of Hill's equation associated with Dirichlet boundary conditions.

Theorem 1.1. [6] If the boundary value problem

$$
\left\{\begin{array}{l}
y^{\prime \prime}(t)+p(t) y(t)=0, \quad a<t<b \\
y(a)=0, \quad y(b)=0
\end{array}\right.
$$

has a nontrivial solution, where $p:[a, b] \rightarrow \mathbf{R}$ is a continuous function, then

$$
\int_{a}^{b}|p(s)| d s>\frac{4}{(b-a)} .
$$

The Lyapunov inequality is useful in the study of spectral properties of ordinary differential equations. It can also be used to establish sufficient conditions on the existence of solutions for certain non-homogeneous boundary value problems, study the zeros of solutions, and obtain bounds on eigenvalues in certain eigenvalue problems. Due to its importance, the Lyapunov inequality has been generalized in many forms. For more details on Lyapunov-type inequalities and their applications, we refer $[1,8,9$, $11,12,13]$ and the references therein.

On the other hand, many researchers have derived Lyapunov-type inequalities for various classes of fractional boundary value problems during the past six years. For the first time, in 2013, Ferreira [3] generalized Theorem 1.1 to the case where the classical second-order derivative in BVP is replaced by an $\alpha^{\text {th }}$-order $(1<\alpha \leq 2)$ Riemann-Liouville type derivative.

Theorem 1.2. [3] If the fractional boundary value problem

$$
\left\{\begin{array}{l}
D_{a}^{\alpha} y(t)+p(t) y(t)=0, \quad a<t<b \\
y(a)=0, \quad y(b)=0,
\end{array}\right.
$$

has a nontrivial solution, where $p:[a, b] \rightarrow \mathbf{R}$ is a continuous function, then

$$
\int_{a}^{b}|p(s)| d s>\Gamma(\alpha)\left(\frac{4}{b-a}\right)^{\alpha-1} .
$$


Here $D_{a}^{\alpha}$ denotes the Riemann-Liouville type $\alpha^{\text {th }}$-order differential operator. In 2014, Ferreira [4] replaced the Riemann-Liouville type derivative in Theorem 1.2 with the Caputo one ${ }^{C} D_{a}^{\alpha}$ and obtained the following Lyapunov-type inequality for the resulting problem:

Theorem 1.3. [4] If the fractional boundary value problem

$$
\left\{\begin{array}{l}
{ }^{C} D_{a}^{\alpha} y(t)+p(t) y(t)=0, \quad a<t<b \\
y(a)=0, \quad y(b)=0
\end{array}\right.
$$

has a nontrivial solution, where $p:[a, b] \rightarrow \mathbf{R}$ is a continuous function, then

$$
\int_{a}^{b}|p(s)| d s>\frac{\Gamma(\alpha) \alpha^{\alpha}}{[(\alpha-1)(b-a)]^{\alpha-1}}
$$

Recently, Ntouyas et al. [7] presented a survey of results on Lyapunovtype inequalities for fractional differential equations associated with a variety of boundary conditions. This article shows that a Lyapunov-type inequality for a two-point Riemann-Liouville type fractional boundary value problem associated with anti-periodic boundary conditions is not yet reported.

In 2016, Dhar et al. [2] derived Lyapunov-type inequalities for two-point Riemann-Liouville type fractional boundary value problems associated with fractional integral boundary conditions. This article stresses the importance of choosing well-posed boundary conditions for Riemann-Liouville type fractional boundary value problems.

Motivated by these developments, in this article, we establish a Lyapunovtype inequality for two-point Riemann-Liouville type fractional boundary value problems associated with well-posed anti-periodic boundary conditions.

\section{Preliminaries}

Throughout, we shall use the following notations, definitions and known results of fractional calculus $[5,10]$. Denote the set of all real numbers and complex numbers by $\mathbf{R}$ and $\mathbf{C}$, respectively. 
Definition 2.1. [5] The Euler gamma function is defined by

$$
\Gamma(z):=\int_{0}^{\infty} t^{z-1} e^{-t} d t, \quad \Re(z)>0 .
$$

Using the reduction formula

$$
\Gamma(z+1)=z \Gamma(z), \quad \Re(z)>0,
$$

the Euler gamma function can be extended to the half-plane $\Re(z) \leq 0$ except for $z=0,-1,-2, \ldots$

Definition 2.2. [5] Let $\alpha>0$ and $a \in \mathbf{R}$. The $\alpha^{\text {th }}$-order RiemannLiouville fractional integral of a function $y:[a, b] \rightarrow \mathbf{R}$ is defined by

$$
I_{a}^{\alpha} y(t)=\frac{1}{\Gamma(\alpha)} \int_{a}^{t}(t-s)^{\alpha-1} y(s) d s, \quad a \leq t \leq b,
$$

provided the right-hand side exists. For $\alpha=0$, define $I_{a}^{\alpha}$ to be the identity map. Moreover, let $n$ denote a positive integer and assume $n-1<\alpha \leq n$. The $\alpha^{\text {th }}$-order Riemann-Liouville fractional derivative is defined as

$$
D_{a}^{\alpha} y(t)=D^{n} I_{a}^{n-\alpha} y(t), \quad a \leq t \leq b,
$$

where $D^{n}$ denotes the classical $n^{\text {th }}$-order derivative, if the right-hand side exists.

Definition 2.3. [5] We denote by $L(a, b)$ the space of Lebesgue measurable functions $y:[a, b] \rightarrow \mathbf{R}$ for which

$$
\|y\|_{L}=\int_{a}^{b}|y(t)| d t<\infty .
$$

Definition 2.4. [5] We denote by $C[a, b]$ the space of continuous functions $y:[a, b] \rightarrow \mathbf{R}$ with the norm

$$
\|y\|_{C}=\max _{t \in[a, b]}|y(t)| .
$$

Definition 2.5. [5] Let $0 \leq \gamma<1, y:(a, b] \rightarrow \mathbf{R}$ and define $y_{\gamma}(t)=$ $(t-a)^{\gamma} y(t), t \in[a, b]$. We denote by $C_{\gamma}[a, b]$ the weighted space of functions $y$ such that $y_{\gamma} \in[a, b]$, and

$$
\|y\|_{C_{\gamma}}=\mid\left(\operatorname{tmaxa}_{t \in[a, b]}^{\gamma} y(t) \mid .\right.
$$


Lemma 2.1. [5] If $\alpha \geq 0$ and $\beta>0$, then

$$
\begin{aligned}
& I_{a}^{\alpha}(t-a)^{\beta-1}=\frac{\Gamma(\beta)}{\Gamma(\beta+\alpha)}(t-a)^{\beta+\alpha-1}, \\
& D_{a}^{\alpha}(t-a)^{\beta-1}=\frac{\Gamma(\beta)}{\Gamma(\beta-\alpha)}(t-a)^{\beta-\alpha-1} .
\end{aligned}
$$

Lemma 2.2. [5] Let $\alpha>\beta>0$ and $y \in C[a, b]$. Then,

$$
D_{a}^{\beta} I_{a}^{\alpha} y(t)=I_{a}^{\alpha-\beta} y(t), \quad t \in[a, b] .
$$

Lemma 2.3. [10] Let $\alpha>0$ and $n$ be a positive integer such that $n-1<$ $\alpha \leq n$. If $y \in C(a, b) \cap L(a, b)$, then the unique solution of the fractional differential equation

$$
D_{a}^{\alpha} y(t)=0, \quad a<t<b,
$$

is

$$
y(t)=C_{1}(t-a)^{\alpha-1}+C_{2}(t-a)^{\alpha-2}+\cdots+C_{n}(t-a)^{\alpha-n},
$$

where $C_{i} \in \mathbf{R}, i=1,2, \cdots, n$.

Lemma 2.4. [10] Let $\alpha>0$ and $n$ be a positive integer such that $n-1<$ $\alpha \leq n$. If $y \in C(a, b) \cap L(a, b)$, then

$$
I_{a}^{\alpha} D_{a}^{\alpha} y(t)=y(t)+C_{1}(t-a)^{\alpha-1}+C_{2}(t-a)^{\alpha-2}+\cdots+C_{n}(t-a)^{\alpha-n},
$$

for some $C_{i} \in \mathbf{R}, i=1,2, \cdots, n$.

\section{Main Results}

In this section, we obtain a Lyapunov-type inequality for an anti-periodic fractional boundary value problem using the properties of the corresponding Green's function.

Theorem 3.1. Let $1<\alpha \leq 2$ and $h:[0, T] \rightarrow \mathbf{R}$. The fractional boundary value problem

(3.1) $\left\{\begin{array}{l}\left(D_{0}^{\alpha} y\right)(t)+h(t)=0, \quad 0<t<T, \\ \left(I_{0}^{2-\alpha} y\right)(0)+\left(I_{0}^{2-\alpha} y\right)(T)=0,\left(D_{0}^{\alpha-1} y\right)(0)+\left(D_{0}^{\alpha-1} y\right)(T)=0,\end{array}\right.$

has the unique solution

$$
y(t)=\int_{0}^{T} G(t, s) h(s) d s, 0<t \leq T
$$


where

$$
G(t, s)=\left\{\begin{array}{lc}
\frac{t^{\alpha-1}}{2 \Gamma(\alpha)}+\frac{t^{\alpha-2}(T-2 s)}{4 \Gamma(\alpha-1)}, & 0<t \leq s \leq T \\
\frac{t^{\alpha-1}}{2 \Gamma(\alpha)}+\frac{t^{\alpha-2}(T-2 s)}{4 \Gamma(\alpha-1)}-\frac{(t-s)^{\alpha-1}}{\Gamma(\alpha)}, & 0<s \leq t \leq T
\end{array}\right.
$$

Proof. Applying $I_{0}^{\alpha}$ on both sides of (3.1) and using Lemma 2.4, we have

$$
y(t)=-\left(I_{0}^{\alpha} h\right)(t)+C_{1} t^{\alpha-1}+C_{2} t^{\alpha-2},
$$

for some $C_{1}, C_{2} \in \mathbf{R}$. Applying $I_{0}^{2-\alpha}$ on both sides of (3.4) and using Lemma 2.1 and Lemma 2.2, we get

$$
\left(I_{0}^{2-\alpha} y\right)(t)=-\int_{0}^{t}(t-s) h(s) d s+C_{1} \Gamma(\alpha) t+C_{2} \Gamma(\alpha-1) .
$$

Applying $D_{0}^{\alpha-1}$ on both sides of (3.4) and using Lemma 2.1 and Lemma 2.2 , we get

$$
\left(D_{0}^{\alpha-1} y\right)(t)=-\int_{0}^{t} h(s) d s+C_{1} \Gamma(\alpha) .
$$

Using $\left(I_{0}^{2-\alpha} y\right)(0)+\left(I_{0}^{2-\alpha} y\right)(T)=0$ in $(3.5)$, we get

$$
\int_{0}^{T}(T-s) h(s) d s=C_{1} \Gamma(\alpha) T+2 C_{2} \Gamma(\alpha-1) .
$$

Using $\left(D_{0}^{\alpha-1} y\right)(0)+\left(D_{0}^{\alpha-1} y\right)(T)=0$ in (3.6), we get

$$
\int_{0}^{T} h(s) d s=2 C_{1} \Gamma(\alpha) .
$$

Solving (3.7) and (3.8) for $C_{1}$ and $C_{2}$, we have

$$
C_{1}=\frac{1}{2 \Gamma(\alpha)} \int_{0}^{T} h(s) d s
$$

and

$$
C_{2}=\frac{1}{2 \Gamma(\alpha-1)} \int_{0}^{T}\left(\frac{T}{2}-s\right) h(s) d s .
$$

Substituting $C_{1}$ and $C_{2}$ in (3.4), we obtain the unique solution of (3.1) as 


$$
\begin{aligned}
y(t) & =-\frac{1}{\Gamma(\alpha)} \int_{0}^{t}(t-s)^{\alpha-1} h(s) d s+\frac{t^{\alpha-1}}{2 \Gamma(\alpha)} \int_{0}^{T} h(s) d s \\
& +\frac{t^{\alpha-2}}{2 \Gamma(\alpha-1)} \int_{0}^{T}\left(\frac{T}{2}-s\right) h(s) d s \\
& =\int_{0}^{t}\left[\frac{t^{\alpha-1}}{2 \Gamma(\alpha)}+\frac{t^{\alpha-2}(T-2 s)}{4 \Gamma(\alpha-1)}-\frac{(t-s)^{\alpha-1}}{\Gamma(\alpha)}\right] h(s) d s \\
& +\int_{t}^{T}\left[\frac{t^{\alpha-1}}{2 \Gamma(\alpha)}+\frac{t^{\alpha-2}(T-2 s)}{4 \Gamma(\alpha-1)}\right] h(s) d s \\
& =\int_{0}^{T} G(t, s) h(s) d s .
\end{aligned}
$$

The proof is complete.

Now, we obtain an upper bound for the Green's function.

Theorem 3.2. For the Green's function $G(t, s)$ defined in (3.3), we observe that

$$
\left|t^{2-\alpha} G(t, s)\right| \leq \frac{(3-\alpha) T}{4 \Gamma(\alpha)}, \forall(t, s) \in[0, T] \times[0, T] .
$$

Proof. Let us consider

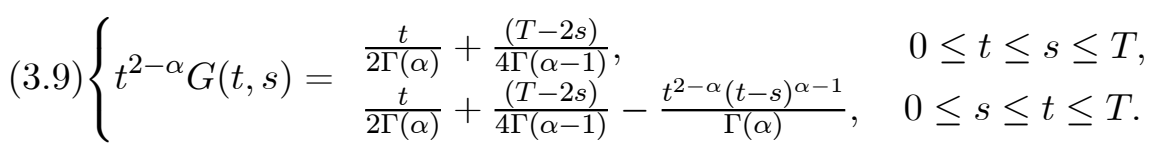

Let us denote the functions

$$
G_{1}(t, s)=\frac{t}{2 \Gamma(\alpha)}+\frac{(T-2 s)}{4 \Gamma(\alpha-1)}
$$

and

$$
G_{2}(t, s)=\frac{t}{2 \Gamma(\alpha)}+\frac{(T-2 s)}{4 \Gamma(\alpha-1)}-\frac{t^{2-\alpha}(t-s)^{\alpha-1}}{\Gamma(\alpha)} .
$$

For a fixed $s \in[0, T]$, we have

$$
\frac{d}{d t} G_{1}(t, s)=\frac{1}{2 \Gamma(\alpha)}>0,
$$

implying that $G_{1}(t, s)$ is an increasing function of $t$. Thus, we have,

$$
\max _{t \in[0, s]}\left|G_{1}(t, s)\right|=\max \left\{\left|G_{1}(0, s)\right|,\left|G_{1}(s, s)\right|\right\} .
$$

We observe that $G_{1}(s, s)$ is an increasing function of $s$, since

$$
\frac{d}{d s} G_{1}(s, s)=\frac{2-\alpha}{2 \Gamma(\alpha)}>0 .
$$


Therefore, we have

$$
\begin{aligned}
\max _{s \in[0, T]} G_{1}(s, s) & =\max \left\{\left|G_{1}(0,0)\right|,\left|G_{1}(T, T)\right|\right\} \\
& =\max \left\{\frac{T}{4 \Gamma(\alpha-1)}, \frac{(3-\alpha) T}{4 \Gamma(\alpha)}\right\} \\
& =\frac{(3-\alpha) T}{4 \Gamma(\alpha)} .
\end{aligned}
$$

Now, we consider

$$
\frac{d}{d s} G_{1}(0, s)=\frac{-1}{2 \Gamma(\alpha-1)}<0 .
$$

So, $G_{1}(0, s)$ is a decreasing function of $s$. Thus, we have

$$
\begin{aligned}
\max _{s \in[0, T]}\left|G_{1}(0, s)\right| & =\max \left\{\left|G_{1}(0,0)\right|,\left|G_{1}(0, T)\right|\right\} \\
& =\max \left\{\frac{T}{4 \Gamma(\alpha-1)}, \frac{T}{4 \Gamma(\alpha-1)}\right\} \\
& =\frac{T}{4 \Gamma(\alpha-1)} .
\end{aligned}
$$

Hence, we have

$$
\max _{t \in[0, s], s \in[0, T]}\left|G_{1}(t, s)\right|=\frac{(3-\alpha) T}{4 \Gamma(\alpha)} .
$$

For a fixed $t \in[0, T]$, we have

$$
\frac{d}{d s} G_{2}(t, s)=\frac{(\alpha-1)(t-s)^{\alpha-2} t^{2-\alpha}}{\Gamma(\alpha)}-\frac{2}{4 \Gamma(\alpha-1)},
$$

and

$$
\frac{d^{2}}{d s^{2}} G_{2}(t, s)=\frac{-(\alpha-1)(\alpha-2)(t-s)^{\alpha-3} t^{2-\alpha}}{\Gamma(\alpha)} \geq 0,
$$

for $s \in[0, t]$. So, $\frac{d}{d s} G_{2}(t, s)$ is an increasing function of $s$ and

$$
\frac{d}{d s} G_{2}(t, t)=\frac{-1}{2 \Gamma(\alpha-1)}<0,
$$

implying that $G_{2}(t, s)$ is a decreasing function of $s$. So,

$$
\max _{s \in[0, t]}\left|G_{2}(t, s)\right|=\max \left\{\left|G_{2}(t, 0)\right|,\left|G_{2}(t, t)\right|\right\} .
$$

Since $\left|G_{2}(t, t)\right|=\left|G_{1}(t, t)\right|$ for $t \in[0, T]$, we only consider $G_{2}(t, 0)$. Now,

$$
\frac{d}{d t} G_{2}(t, 0)=\frac{-1}{2 \Gamma(\alpha)}<0,
$$


implying that $G_{2}(t, 0)$ is a decreasing function of $t$. So, we have

Therefore,

$$
\begin{aligned}
\max _{t \in[0, T]}\left|G_{2}(t, 0)\right| & =\max \left\{\left|G_{2}(0,0)\right|,\left|G_{2}(T, 0)\right|\right\} \\
& =\max \left\{\frac{T}{4 \Gamma(\alpha-1)}, \frac{(3-\alpha) T}{4 \Gamma(\alpha)}\right\} \\
& =\frac{(3-\alpha) T}{4 \Gamma(\alpha)} .
\end{aligned}
$$

$$
\max _{s \in[0, t], t \in[0, T]}\left|G_{2}(t, s)\right|=\frac{(3-\alpha) T}{4 \Gamma(\alpha)} .
$$

Hence, the result follows from (3.10) and (3.11).

We are now able to formulate a Lyapunov-type inequality for a RiemannLiouville type fractional boundary value problem with anti-periodic boundary conditions.

Theorem 3.3. If the following fractional boundary value problem

$$
\left\{\begin{array}{l}
\left(D_{0}^{\alpha} y\right)(t)+p(t) y(t)=0, \quad 0<t<T \\
\left(I_{0}^{2-\alpha} y\right)(0)+\left(I_{0}^{2-\alpha} y\right)(T)=0,\left(D_{0}^{\alpha-1} y\right)(0)+\left(D_{0}^{\alpha-1} y\right)(T)=0
\end{array}\right.
$$

has a nontrivial solution, then

$$
\int_{0}^{T} s^{\alpha-2}|p(s)| d s>\frac{4 \Gamma(\alpha)}{(3-\alpha) T} .
$$

Proof. Let $B=C_{2-\alpha}[0, T]$ be the Banach space of functions $y$ endowed with norm

$$
\|y\|_{C_{2-\alpha}}=\max _{t \in[0, T]}\left|t^{2-\alpha} y(t)\right| .
$$

It follows from Theorem (3.12) that a solution to (3.12) satisfies the equation

$$
y(t)=\int_{0}^{T} G(t, s) p(s) y(s) d s
$$

Hence,

$$
\begin{aligned}
\|y\|_{C_{2-\alpha}} & =\max _{t \in[0, T]}\left|t^{2-\alpha} \int_{0}^{T} G(t, s) p(s) y(s) d s\right| \\
& \leq \max _{t \in[0, T]}\left[\int_{0}^{T}\left|t^{2-\alpha} G(t, s)\right||p(s)||y(s)| d s\right] \\
& \leq\|y\|_{C_{2-\alpha}}\left[\max _{t \in[0, T]} \int_{0}^{T}\left|t^{2-\alpha} G(t, s)\right| s^{\alpha-2}|p(s)| d s\right] \\
& \leq\|y\|_{C_{2-\alpha}}\left[\max _{t \in[0, T]}\left|t^{2-\alpha} G(t, s)\right|\right] \int_{0}^{T} s^{\alpha-2}|p(s)| d s
\end{aligned}
$$


or, equivalently,

$$
1<\left[\max _{t \in[0, T]}\left|t^{2-\alpha} G(t, s)\right|\right] \int_{0}^{T} s^{\alpha-2}|p(s)| d s .
$$

An application of Theorem 3.2 yields the result.

\section{Application}

In this section, we estimate a lower bound for the eigenvalue of the fractional eigenvalue problem corresponding to (3.12).

Theorem 4.1. Assume that $y$ is a nontrivial solution of the fractional eigenvalue problem

(4.1) $\left\{\begin{array}{l}\left(D_{0}^{\alpha} y\right)(t)+\lambda y(t)=0, \quad 0<t<T, \\ \left(I_{0}^{2-\alpha} y\right)(0)+\left(I_{0}^{2-\alpha} y\right)(T)=0,\left(D_{0}^{\alpha-1} y\right)(0)+\left(D_{0}^{\alpha-1} y\right)(T)=0,\end{array}\right.$

where $y(t) \neq 0$ for each $t \in(0, T)$. Then,

$$
|\lambda|>\frac{4 \Gamma(\alpha)(\alpha-1)}{T^{\alpha}(3-\alpha)}, \quad 1<\alpha \leq 2 .
$$

Proof. From (3.13), we obtain

$$
\int_{0}^{T} s^{\alpha-2}|\lambda| d s>\frac{4 \Gamma(\alpha)}{(3-\alpha) T}
$$

or, equivalently,

$$
|\lambda|>\frac{4 \Gamma(\alpha)(\alpha-1)}{T^{\alpha}(3-\alpha)} .
$$

This proves the result (4.2). The proof is complete. 


\section{References}

[1] R. C. Brown and D. B. Hinton, "Lyapunov Inequalities and their applications," in Survey on classical inequalities, T. M. Rassias, Ed. Dordrecht: Springer, 2000, pp. 1-25. doi: 10.1007/ 978-94-011-4339-4_1

[2] S. Dhar, Q. Kong, and M. Mccabe, "Fractional boundary value problems and Lyapunov-type inequalities with fractional integral boundary conditions", Electronic journal of qualitative theory of differential equations, vol. 2016, Art. ID. 43, 2016. doi: 10.14232/ ejqtde.2016.1.43

[3] R. A. C. Ferreira, "A Lyapunov-type inequality for a fractional boundary value problem", Fractional calculus and applied analysis, vol. 16, no. 4, pp. 978-984, 2013. doi: 10.2478/ s13540-013-0060-5

[4] R. A. C. Ferreira, "On a Lyapunov-type inequality and the zeros of a certain Mittag-Leffler function", Journal of mathematical analysis and applications, vol. 412, no. 2, pp. 1058-1063, 2014. doi: 10.1016/ j.jmaa.2013.11.025

[5] A. A. Kilbas, H. M. Srivastava and J.J. Trujillo, Theory and applications of fractional differential equations. Amsterdam: Elsevier, 2006.

[6] A. Liapounoff, "Problème général de la stabilité du mouvement", Annales de la Faculté des sciences de l'Université de Toulouse pour les sciences mathématiques et les sciences physiques, vol. 9, pp. 203-474, 1907. [On line]. Available: https:/ / bit.ly/ 3hCGZTk

[7] S. K. Ntouyas, B. Ahmad and T.P. Horikis, "Recent developments of Lyapunov-type inequalities for fractional differential equations", 28 Apr 2018, arXiv:1804.10760v1

[8] B. G. Pachpatte, "On Lyapunov type inequalities for certain higher order differential equations", Journal of mathematical analysis and applications, vol. 195, no. 2, pp. 527-536, 1995 . doi: 10.1006/jmaa.1995.1372

[9] J. P. Pinasco, Lyapunov-type inequalities with applications to eigenvalue problems. New York (NY): Springer, 2013. doi: 10.1007/978-1-46148523-0 
[10] I. Podlubny, Fractional differential equations. San Diego (CA): Academic Press, 1999.

[11] A. Tiryaki, "Recent developments of Lyapunov-type inequalities", Advances in dynamical systems and applications, vol. 5, no. 2, pp. 231-248, 2010. [On line]. Available: https:/ / bit.ly/ 2U6mMfB

[12] X. Yang, Y. Kim and K. Lo, "Lyapunov-type inequality for a class of even-order linear differential equations", Applied mathematics and computation, vol. 245, pp. 145-151, 2014. doi: 10.1016/j.amc. 2014.07.085

[13] X. Yang, Y. Kim and K. Lo, "Lyapunov-type inequalities for a class of higher-order linear differential equations", Applied mathematics letters, vol. 34, pp. 86-89, 2014. doi: 10.1016/j.aml.2013.11.001 